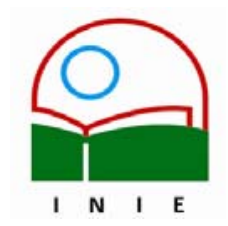

Universidad de Costa Rica

Facultad de Educación

Instituto de Investigación en Educación

ACTUALDADES INVESTIG ATIVASEN EDUCACION

\title{
DESARROLLO Y APLICACIÓN DE LECCIONES DE ESTADÍSTICA EN-LÍNEA CON OBJETOS DE APRENDIZAJE EN UN AMBIENTE UNIVERSITARIO
}

\author{
PEDAGOGICAL IMPACT OF CONSTRUCTIVIST LEARNING OBJECTS \\ IN STATISTIC TEACHING
}

\begin{abstract}
Javier Organista Sandoval ${ }^{1}$ Gilles Lavigne ${ }^{2}$

Resumen: Se presentan resultados preliminares de una investigación-desarrollo que tuvo el propósito de desarrollar lecciones de Estadística en línea con inserción de objetos de aprendizaje y estimar el efecto sobre el aprendizaje de los alumnos participantes en un ambiente natural universitario. Se utilizaron señalamientos teóricos constructivistas para el desarrollo de las lecciones, para conformarle al estudiante un ambiente en línea propicio para la construcción activa de su conocimiento.

La aplicación de las lecciones se realizó con 92 alumnos universitarios (tres grupos). Se aplicó una encuesta de datos generales previo a la intervención educativa. Al final, se entrevistaron a los docentes responsables y se conformaron tres grupos de discusión con los alumnos. Los resultados mostraron un mejor aprendizaje en Estadística en 6 de las 7 comparaciones hechas para los subgrupos que tuvieron la mayor actividad en el web. La opinión de alumnos y docentes fue favorable a este tipo de innovación educativa. Finalmente, es conveniente puntualizar las bondades de incorporar este tipo de innovaciones en cursos universitarios y asi transitar hacia una mejora integral del proceso educativo.
\end{abstract}

Palabras claves: DIDÁCTICA DE ESTADÍSTICA; OBJETOS DE APRENDIZAJE EN ESTADÍSTICA; INNOVACIÓN DE LA ENSEÑANZA.

Abstract: This communication is presenting the preliminary results of a research-development project aiming to evaluate the pedagogical impact of constructivist based learning objects integrated into online Statistic lessons within a live university environment. The experiment was performed with 92 students distributed into three course groups. A survey of the students' general profile was applied previous to the educative intervention and, thereafter, the three participating teachers were interviewed and the students invited to participate to three discussion groups. The results show that, with the exception of one subgroup, the students with a greater online activity obtained a better mark. The overall opinion of the teachers and of the students was favorable to this type of education innovation. This standpoint should give support to the widening of this type of innovation to a broader range of university programs, towards an integral improvement of the educative process.

Key words: DIDACTICS OF STATISTIC; LEARNING OBJECTS IN STATISTIC; INNOVATION OF EDUCATION.

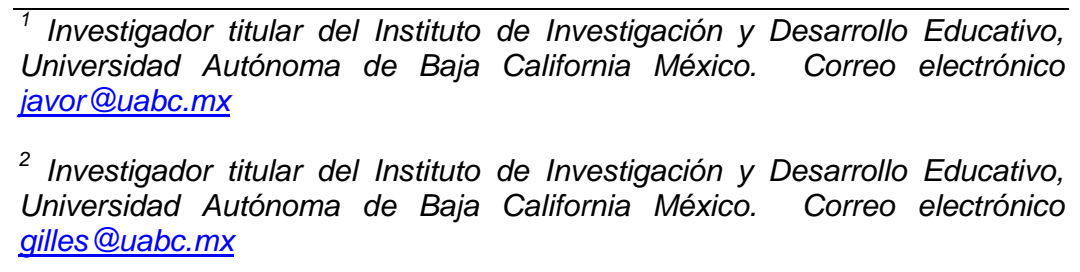

Artículo recibido: 11 de setiembre, 2006

Aprobado: 13 de diciembre, 2006 


\section{Introducción}

Algunos autores (Simonson, Smaldino, Albright y Zvacek, 2003) coinciden en señalar que en las dos últimas décadas la sociedad se ha visto influenciada por el gran desarrollo de las llamadas tecnologías de la información y la comunicación. En gran medida, los cambios tecnológicos han estado asociados a la evolución de los sistemas de cómputo y de telecomunicaciones. Así, se ha logrado establecer una gran red a nivel mundial conocida como Internet, la cual representa una fuerza potencial ${ }^{3}$ de 1043 millones de usuarios para la entrega de contenidos educativos bajo diferentes medios y facilitar un aprendizaje de calidad.

En el área educativa, la incorporación de las llamadas nuevas tecnologías ha propiciado un replanteamiento de los métodos de enseñanza y de aprendizaje. Para el caso específico de la llamada 'ciencia de los datos', como se le conoce a Estadística, las nuevas tecnologías de la información y la comunicación han acelerado el avance hacia un enfoque constructivista en su enseñanza; o como señala de forma más puntual Batanero (2001), la enseñanza de la Estadística puede propiciarse mediante la consideración de estrategias constructivistas específicas, tales como: la interacción con objetos, la resolución de problemas, la interacción social y cultural o mediante una recopilación de las anteriores.

Por otro lado, el desarrollo tecnológico también ha influido para que se establezcan propuestas para organizar de mejor manera la abundante información que actualmente existe en el web. Así ha surgido una tendencia en crear entidades de información que puedan ser reutilizables al momento de desarrollar materiales educativos. Considerando lo anterior, es conveniente señalar la necesidad de transitar hacia una innovación integral, que aborde aspectos tanto de fondo como de forma. Es decir, que recurra a las aportaciones teóricas principales durante la construcción de los materiales educativos y por otra parte, que considere aspectos organizacionales de la información.

${ }^{3}$ Fuente: Nielsen//NetRatings (http://www.nielsen-netratings.com/). 30 junio de 2006. 


\section{Referentes teóricos}

La organización de la información, estrategia básica para el desarrollo de sistemas educativos vía Internet, también se ha visto influenciada por la velocidad de los cambios tecnológicos, de tal manera que en los últimos cinco años, ha cobrado auge una tendencia en el campo de la tecnología educativa relacionada con el desarrollo e incorporación de objetos de aprendizaje (Murphy, 2004).

Los objetos de aprendizaje son considerados una herramienta educativa muy importante, que pueden insertarse en propuestas curriculares y metodologías de enseñanza-aprendizaje de diversa índole. En muchos casos, son vistos como una estrategia de innovación educativa. Una de las definiciones más difundidas, plantea al objeto de aprendizaje como cualquier recurso digital que puede ser reutilizado como soporte para el aprendizaje (Wiley, 2001); o como señala Merrill (2002), un objeto de aprendizaje es un objeto mediático conjunto de bits de texto, gráficos, video o audio- al cual se le integra una estrategia instruccional.

Por otro lado, surge la interrogante de ¿cuál sería la didáctica más adecuada para la enseñanza de la Estadística ante una influencia creciente de las tecnologías de la información y comunicación, especialmente del desarrollo de la tecnología de Internet?

Al respecto, Smith-Gratto (2000) sostiene que para diseñar un ambiente de aprendizaje constructivista en el web se debe ofrecer de: i) nuevas experiencias que no se ajusten al esquema actual del estudiante; ii) actividades que ayuden al estudiante a reestructurar su comprensión; iii) actividades de solución a problemas contextuales y iv) actividades que requieran interacción social.

Con base en lo anterior, y dado que no se disponen de estudios formales en México que aborden la didáctica de la Estadística bajo un enfoque constructivista, la presente investigación propone un desarrollo tecnológico centrado en implementar lecciones para la enseñanza en línea de la Estadística, con un enfoque constructivista y una organización de la información basada en objetos de aprendizaje. La parte de investigación se ubica en el campo educativo, específicamente en evaluar el aprendizaje adquirido por los estudiantes ante la innovación educativa mencionada y en conocer la opinión de los usuarios de las lecciones. Específicamente, se pretende dar respuesta a la interrogante: 
¿Es posible mejorar el aprendizaje de los alumnos en Estadística con el uso de lecciones que incluyan objetos de aprendizaje y estrategias constructivistas?

\section{Objetivos}

Estimar el efecto sobre el aprendizaje de los alumnos de tres cursos de Estadística básica, que resulta de aplicar lecciones de Estadística en línea con inserción de objetos de aprendizaje y estrategias constructvistas en un ambiente natural universitario.

\section{Método}

Un punto fundamental en el diseño de investigación y desarrollo (Charles, 1988), enfoque donde se ubica el presente estudio, es la investigación del efecto que tiene el producto o desarrollo generado. Por ello, en esta sección primeramente se describe el procedimiento utilizado para desarrollar las lecciones; y en un segundo apartado se aborda la forma como se realizó la investigación en un ambiente natural universitario.

\subsection{Desarrollo de las lecciones}

i) Selección de contenidos. Se revisaron las cartas descriptivas de los cursos de Estadística del tronco común en ciencias sociales de la Facultad de Ciencias Administrativas y Sociales y dos programas de Maestría de la Universidad Autónoma de Baja California (UABC). La intención fue seleccionar aquellos temas estadísticos que fueran comunes y así contar con un potencial mayor de posibles participantes. Como resultado de la revisión a las cartas descriptivas, se seleccionaron tres cursos de Estadística básica, cuyos docentes colaboraron en la selección, delimitación y extensión de los contenidos. Como resultado de dichas opiniones, se decidió considerar los temas: i) Curva normal, ii) Hipótesis y iii) Contrastes Z y t-student.

ii) Organización de la información. A partir de los contenidos definidos, el desarrollo de las lecciones considera las sugerencias de Mohan (2004) con relacion a la utilización de objetos de aprendizaje que se articulan para dar forma a tres lecciones de Estadística (ver figura 1). Según Roig-Vila (2005), el planteamiento basado en objetos de aprendizaje es, actualmente, uno de los pilares del aprendizaje a través de Internet. En palabras de Hodgins (2000), los objetos de aprendizaje están destinados a cambiar para siempre la forma y el fondo del aprendizaje. 


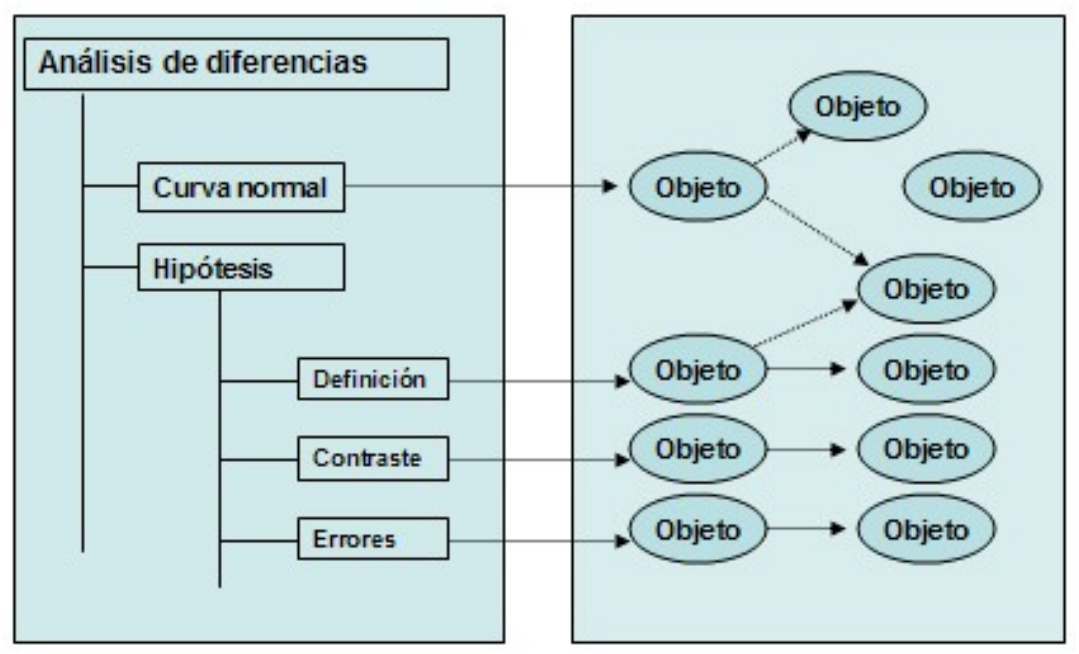

Figura 1. Ejemplo de objetos y sus posibles relaciones con un tema

iii) Didáctica utilizada. La didáctica utilizada tuvo sustento en la combinación de la tecnología actual, especialmente de software libre, con los desarrollos teóricos existentes tales como el constructivismo, específicamente las aportaciones piagetanas de la construcción activa del conocimiento (Leflore, 2000), la resolución de problemas en un contexto real y el aprendizaje en comunidad. Con base en lo anterior, la didáctica utilizada propone la interacción del participante con los objetos de aprendizaje, la resolución de una serie de problemas contextualizados al área educativa y la inclusión de foros de discusión asíncronos, para el intercambio de ideas, opiniones y asesorías (ver figuras 2a, 2b y 2c). 


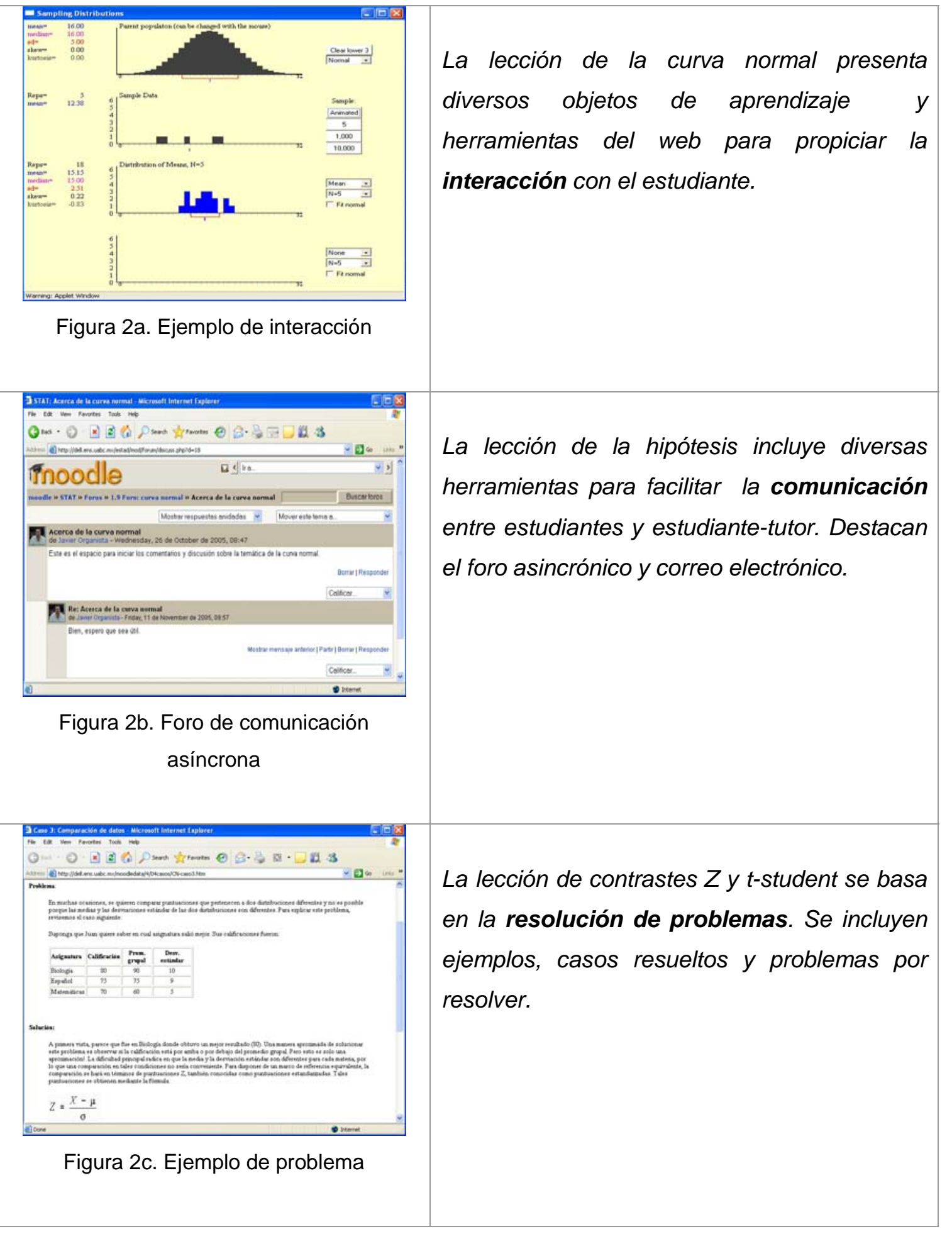


iv) Implementación. Se utilizaron diversas aplicaciones de software libre para lograr desarrollar e instalar las lecciones de Estadística. El ambiente de gestoría de código libre Moodle, fue el que se utilizó para alojar las lecciones de Estadística. Para el desarrollo de los materiales educativos se utilizaron el editor de hipertexto Dreamweaver, Impatica, un editor de imagen y herramientas programáticas del web.

La figura 3 muestra la interfaz para la lección de la curva normal. La organización de la información de cada lección se realizó considerando 10 secciones: 1. Ruta de aprendizaje; 2. Problema guía; 3. Objetivo; 4. Conceptos; 5. Casos resueltos; 6. Herramientas; 7. Información adicional; 8. Contacto; 9. Foro y 10. Evaluación.

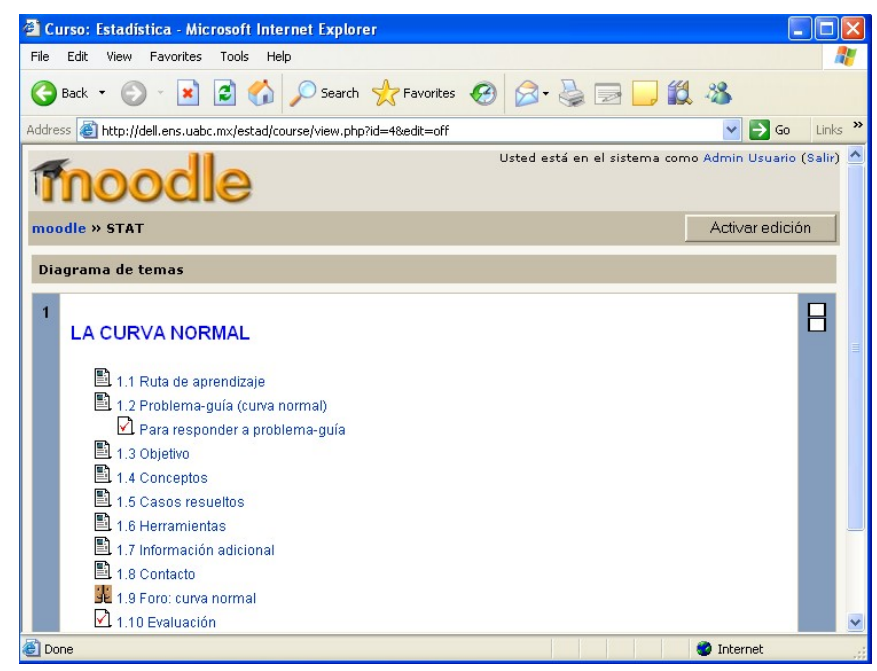

Figura 3. Menú principal de la lección de la curva normal

v) Evaluación preliminar. La evaluación preliminar de las lecciones de Estadística en línea con objetos de aprendizaje se realizó desde la perspectiva de tres docentes y cuatro alumnos, quienes expresaron sus comentarios y sugerencias acerca de los contenidos, de la navegación, de la interfaz y en general de las lecciones. La retroalimentación obtenida permitió liberar una versión más eficiente de las lecciones. 


\subsection{Aplicación de las lecciones}

En esta sección se describe el procedimiento utilizado para aplicar las lecciones en tres cursos formales de Estadística. La evaluación educativa hecha considera aspectos tanto cuantitativos como cualitativos para estimar el rendimiento académico logrado con dichas lecciones. Se incluyen las opiniones de los usuarios participantes.

i) Contexto. La intervención educativa se realizó durante el primer período escolar del 2006 en las instalaciones de la Unidad Universitaria de Valle Dorado de la UABC en la ciudad de Ensenada. Los cursos de Estadística tuvieron duración semestral y caracter obligatorio para los alumnos.

ii) Participantes. Se consideraron los alumnos participantes de tres cursos formales de Estadística. El primer grupo estuvo conformado por 41 alumnos, el segundo por 24 y el tercero de 27. Los 92 alumnos tuvieron como requisito acreditar el curso de Estadística respectivo, como parte de su carga académica semestral. Los docentes responsables de cada grupo, mostraron interés y disposición de apoyar la presente investigación.

\section{iii) Instrumentos.}

a) Lecciones. Constituyen un elemento fundamental en la intervención educativa. Como ya se mencionó, dichas lecciones tuvieron un soporte pedagógico constructivista e incluyeron objetos de aprendizaje.

b) Encuesta de datos generales. Tuvo el propósito de caracterizar los aspectos generales del estudiante, su trayectoria escolar, su percepción sobre el aprendizaje, su actitud hacia la Estadística y su opinión acerca del uso de tecnología. Dichas variables se incluyeron con base en la recomendación de O'Malley (2002), quien sostiene que la percepción de los estudiantes hacia el aprendizaje en línea puede determinarse a partir de los constructos: i) condiciones educativas previas; ii) características de los estudiantes y iii) características percibidas sobre aprendizajes en línea. Los reactivos fueron de opción múltiple, de respuesta abierta y de opción múltiple sobre una Escala de Likert con 5 pasos entre el desacuerdo total y el acuerdo total. 
c) Listado de calificaciones. A cada docente participante se le solicitó una copia de las calificaciones parciales y que indicara la correspondencia entre la calificación parcial del alumno y la temática abordada con la lección.

d) Archivos de registro del sistema. Se incluyó la información generada por la herramienta de tracking de Moodle, para analizar la actividad del usuario con el servidor web que hospedó a las lecciones, específicamente para registrar:

- accesos al sitio web según el usuario

- registros por hora/día/semana

- duración de la sesión

- la actividad y recursos consultados

- errores

e) Guía para el grupo de discusión. Se consideraron tres grupos de discusión con ocho estudiantes cada uno. La selección de los estudiantes fue intencional, cuidando incorporar opiniones diferentes que propiciaran el intercambio de ideas, de conformidad a las sugerencias expuestas por Álvarez-Gayou (2005). El propósito fue conocer la opinión de los estudiantes acerca de las lecciones y del efecto de las mismas sobre el aprendizaje del alumno.

f) Guía para la entrevista. Del tipo semi-estructurada. Se dirigió a tres docentes de la materia de Estadística que participaron y utilizaron las lecciones en línea. La entrevista se dirigió a obtener información acerca de datos generales del maestro, su actitud hacia la Estadística y su opinión acerca de las lecciones.

iv) Procedimiento. Las tres lecciones se aplicaron a los grupos 1 y 2 de conformidad a los tiempos en que el docente responsable de la materia revisaba los temas frente a grupo y de acuerdo a su carta descriptiva respectiva. Para el grupo 3 solo se aplicó la lección de la curva normal, porque así lo indicaba su carta descriptiva. En la etapa I, se aplicó la encuesta de datos generales a los tres grupos; la etapa II corresponde al período de la intervención. Finalmente, en la etapa III se realizaron las entrevistas y los grupos de discusión (ver tabla I). 
Tabla I. Diseño utilizado para aplicar las lecciones a los grupos.

\begin{tabular}{|c|c|c|c|c|}
\hline ETAPA I & \multicolumn{3}{|c|}{ ETAPA II } & ETAPA III \\
\hline & $\begin{array}{c}\text { Curva } \\
\text { normal }\end{array}$ & Hipótesis & $\begin{array}{c}\text { Contrastes } \\
\text { Z y t }\end{array}$ & \\
\hline \multirow{3}{*}{$\begin{array}{l}\text { Encuesta de datos } \\
\text { generales }\end{array}$} & G1* & G1 & G1 & \multirow{3}{*}{$\begin{array}{l}\text { - Gpo. de discusión } \\
\text { - Entrevista }\end{array}$} \\
\hline & G2 & G2 & G2 & \\
\hline & G3 & - & - & \\
\hline
\end{tabular}

v) Análisis de resultados. La información obtenida a partir de la encuesta de datos generales, las calificaciones de los maestros y los registros del sistema se analizaron mediante un enfoque estadístico de tipo descriptivo-exploratorio. El análisis se dirigió a obtener: la estadística descriptiva básica, análisis de frecuencias, gráficas, análisis $t$-student, entre otros. El propósito del análisis fue obtener una caracterización general de los estudiantes, disponer de evidencias del desempeño académico obtenido con la didáctica aquí expuesta. Para procesar la información se utilizó el programa estadístico SPSS ver. 14.0. Para las transcripciones generadas de las entrevistas y grupos de discusión, se efectuó un análisis de contenido para extraer las ideas principales.

\section{5. $\quad$ Resultados}

Los resultados se organizaron en tres apartados. El primero para describir las características generales de los participantes. El segundo apartado muestra la información obtenida acerca del efecto de las lecciones sobre el aprendizaje de los alumnos participantes. El último de los apartados engloba las opiniones expresadas por maestros y alumnos.

\subsection{Características generales de los participantes.}

Con relación al género, solo el grupo II muestra un equilibrio cercano al $50 \%$ mientras que en el grupo I el porcentaje de mujeres alcanza el 68\%, situación que se invierte en el grupo III. El estado civil de los participantes es mayormente soltero ya que en los tres grupos su porcentaje rebasa el $80 \%$. Respecto a la actividad laboral, llama la atención el $62 \%$ de los participantes del grupo I que no labora, mientras que en los otros grupos la mayoría de los estudiantes se encuentra laborando con mayor o menor intensidad. Destaca el $47 \%$ que alcanza la actividad laboral entre 11 y +20 hrs. de los alumnos del grupo II (ver tabla II). 
Tabla II. Características generales de los alumnos participantes

\begin{tabular}{|c|c|c|r|r|r|r|r|}
\hline \multirow{2}{*}{} & \multicolumn{2}{c|}{ Grupo I } & \multicolumn{2}{c|}{ Grupo II } & \multicolumn{2}{c|}{ Grupo III } \\
\cline { 2 - 8 } \multicolumn{2}{|c|}{} & $\mathrm{n}$ & \multicolumn{1}{c|}{$\%$} & $\mathrm{n}$ & \multicolumn{1}{c|}{$\%$} & $\mathrm{n}$ & \multicolumn{1}{c|}{$\%$} \\
\hline \multicolumn{2}{|c|}{ Sujetos } & 37 & 100.0 & 17 & 100.0 & 18 & 100.0 \\
\hline \multirow{2}{*}{ Género } & Hombres & 12 & 32.4 & 8 & 47.1 & 13 & 72.2 \\
\cline { 2 - 8 } & Mujeres & 25 & 67.6 & 9 & 52.9 & 5 & 27.8 \\
\hline \multirow{2}{*}{ Estado civil } & Soltero & 34 & 91.9 & 14 & 82.4 & 18 & 100.0 \\
\cline { 2 - 8 } & Casado & 3 & 8.1 & 3 & 17.6 & 0 & 0 \\
\hline \multirow{3}{*}{$\begin{array}{c}\text { Actividad } \\
\text { laboral }\end{array}$} & 0 hrs. & 23 & 62.2 & 3 & 17.6 & 3 & 16.7 \\
\cline { 2 - 8 } & $1-10$ & 4 & 10.8 & 6 & 35.3 & 3 & 16.7 \\
\cline { 2 - 8 } & $11-20$ & 2 & 5.4 & 3 & 17.6 & 3 & 16.7 \\
\cline { 2 - 8 } & $20+$ & 8 & 21.6 & 5 & 29.4 & 9 & 50.0 \\
\hline \multirow{2}{*}{$\begin{array}{c}\text { Tipo de } \\
\text { bachillerato }\end{array}$} & Público & 28 & 75.7 & 14 & 82.4 & 15 & 83.3 \\
\cline { 2 - 8 } & Privado & 9 & 24.3 & 3 & 17.6 & 3 & 16.7 \\
\hline
\end{tabular}

En la tabla III se presentan las medias de las calificaciones obtenidas por los alumnos en bachillerato y universidad. El grupo II muestra menor desempeño tanto en bachillerato como en universidad. El grupo I alcanza el mejor promedio en universidad mientras que el grupo III lo logra en bachillerato.

Tabla III. Características académicas de los alumnos participantes

\begin{tabular}{|c|c|c|c|c|c|c|}
\hline \multirow{2}{*}{} & \multicolumn{2}{|c|}{ Grupo I } & \multicolumn{2}{c|}{ Grupo II } & \multicolumn{2}{c|}{ Grupo III } \\
\cline { 2 - 7 } & Media & D.E.* & Media & D.E. & Media & D.E. \\
\hline Edad & 21.35 & 3.85 & 23.76 & 6.05 & 21.67 & 3.76 \\
\hline $\begin{array}{c}\text { Promedio en } \\
\text { bachillerato }\end{array}$ & 8.30 & 0.61 & 8.23 & 0.66 & 8.55 & 0.59 \\
\hline $\begin{array}{c}\text { Promedio en } \\
\text { universidad }\end{array}$ & 8.79 & 0.47 & 8.33 & 0.59 & 8.68 & 0.54 \\
\hline
\end{tabular}

\subsection{Efecto de las lecciones sobre el aprendizaje}

Para cada uno de los grupos se hizo una clasificación en subgrupos de acuerdo a la actividad registrada por los usuarios según el sistema de tracking de Moodle (vea tabla IVa, IVb y IVc). Así resultaron los subgrupos de mayor actividad (ActMayor) y menor actividad (ActMenor). A partir de los subgrupos creados, se aplicó la prueba de Levene, conocida también como prueba de homocedasticidad o de igualdad de varianzas. Dicho contraste permite determinar si las varianzas de los subgrupos comparados se pueden considerar como equivalentes. En ningún caso, se detectaron diferencias significativas por lo que los subgrupos se consideraron equivalentes. 
Para indagar si hubo diferencias significativas entre las medias de las calificaciones obtenidas por los alumnos del subgrupo (ActMayor) con relación al de (ActMenor) se aplicó la prueba t-student para muestras independientes. Al nivel de significancia de 0.05 no se presentaron diferencias significativas en ninguno de los contrastes que se muestran en las tablas IVa, IVb y IVc. Sin embargo, Ilama la atención en la figura 4, la tendencia a mejorar el aprendizaje de los participantes de los subgrupos de (ActMayor) en 6 de 7 comparaciones.

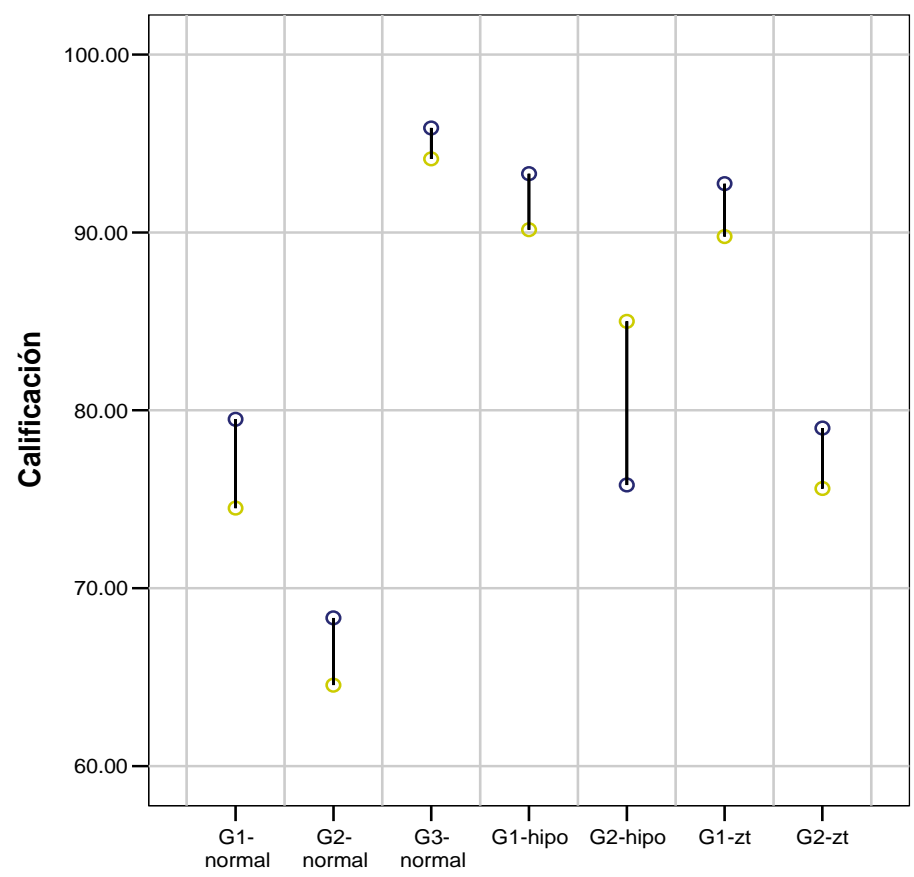

ActMayor

ActMenor

Gpo-tema

Tabla IVa. Descriptivos básicos asociados a las calificaciones obtenidas por los alumnos de los subgrupos de +Activ y-Activ en el tema de la curva normal.

\begin{tabular}{|r|c|c|c|c|c|c|}
\hline \multirow{2}{*}{ CURVA NORMAL } & \multicolumn{2}{|c|}{ Grupo I } & \multicolumn{2}{c|}{ Grupo II } & \multicolumn{2}{c|}{ Grupo III } \\
\cline { 2 - 8 } & + Activ & - & +Activ & -Activ. & +Activ & - \\
Activ. & $\cdot$ & & Activ. \\
\hline No. de participantes & 20 & 20 & 12 & 11 & 15 & 15 \\
\hline $\begin{array}{r}\text { Significancia } \\
\text { (Homocedasticidad) }\end{array}$ & \multicolumn{2}{|c|}{0.77} & \multicolumn{2}{|c|}{0.60} & \multicolumn{2}{|c|}{0.02} \\
\hline Media & 79.50 & 74.50 & 68.33 & 64.55 & 95.87 & 94.13 \\
\hline Desviación estándar & 23.50 & 26.05 & 18.38 & 24.34 & 6.08 & 8.61 \\
\hline Error estándar media & 5.25 & 5.82 & 5.31 & 7.34 & 1.57 & 2.22 \\
\hline Valor t-student* & \multicolumn{2}{|c|}{0.64} & \multicolumn{2}{|c|}{0.42} & \multicolumn{2}{|c|}{0.64} \\
\hline Significancia (t-student) & \multicolumn{2}{|c|}{0.52} & \multicolumn{2}{|c|}{0.68} & \multicolumn{2}{|c|}{0.53} \\
\hline
\end{tabular}

* Prueba de Levene para igualdad de varianzas; significancia $\mathrm{P}<0.05$

** Prueba t-student para muestras independientes 
Tabla IVb. Descriptivos básicos asociados a las calificaciones obtenidas por los alumnos de los subgrupos de +Activ y -Activ en el tema de hipótesis.

\begin{tabular}{|c|c|c|c|c|c|c|}
\hline \multirow[b]{2}{*}{ HIPOTESIS } & \multicolumn{2}{|c|}{ Grupo I } & \multicolumn{2}{|c|}{ Grupo II } & \multicolumn{2}{|c|}{ Grupo III } \\
\hline & $\begin{array}{c}\text { +Activ } \\
\text {. }\end{array}$ & $\begin{array}{c}- \\
\text { Activ. }\end{array}$ & $\begin{array}{c}+ \text { Activ } \\
\text {. }\end{array}$ & -Activ. & +Activ & $\begin{array}{c}- \\
\text { Activ. }\end{array}$ \\
\hline No. de participantes & 20 & 20 & 12 & 11 & - & - \\
\hline $\begin{array}{r}\text { Significancia } \\
\text { (Homocedasticidad)* }\end{array}$ & \multicolumn{2}{|c|}{0.16} & \multicolumn{2}{|c|}{0.02} & \multicolumn{2}{|c|}{-} \\
\hline Media & 93.30 & 90.15 & 75.8 & 85.00 & - & - \\
\hline Desviación estándar & 12.75 & 16.90 & 27.46 & 8.06 & - & - \\
\hline Error estándar media & 2.85 & 3.78 & 7.93 & 2.43 & - & - \\
\hline Valor t-student** & \multicolumn{2}{|c|}{0.67} & \multicolumn{2}{|c|}{-1.11} & \multicolumn{2}{|c|}{ - } \\
\hline Significancia (t-student) & \multicolumn{2}{|c|}{0.51} & \multicolumn{2}{|c|}{0.29} & \multicolumn{2}{|c|}{ - } \\
\hline
\end{tabular}

* Prueba de Levene para igualdad de varianzas; significancia $\mathrm{P}<0.05$

** Prueba t-student para muestras independientes

Tabla IVc. Descriptivos básicos asociados a las calificaciones obtenidas por los alumnos de los subgrupos de +Activ y-Activ en el tema de Z/t-student.

\begin{tabular}{|c|c|c|c|c|c|c|}
\hline \multirow{2}{*}{$\begin{array}{l}\text { CONTRASTES } \\
\text { Z/T-STUDENT }\end{array}$} & \multicolumn{2}{|c|}{ Grupo I } & \multicolumn{2}{|c|}{ Grupo II } & \multicolumn{2}{|c|}{ Grupo III } \\
\hline & $\begin{array}{c}+ \text { Activ } \\
.\end{array}$ & $\begin{array}{c}- \\
\text { Activ. }\end{array}$ & $\begin{array}{c}+ \text { Activ } \\
.\end{array}$ & -Activ. & +Activ & $\begin{array}{c}\text { - } \\
\text { Activ. }\end{array}$ \\
\hline No. de participantes & 20 & 20 & 11 & 10 & - & - \\
\hline $\begin{array}{r}\text { Significancia } \\
\text { (Homocedasticidad)* }\end{array}$ & \multicolumn{2}{|c|}{0.65} & \multicolumn{2}{|c|}{0.92} & & \\
\hline Media & 92.74 & 89.77 & 79.00 & 75.60 & - & - \\
\hline Desviación estándar & 8.32 & 10.29 & 18.88 & 25.11 & - & - \\
\hline Error estándar media & 1.86 & 2.30 & 5.69 & 7.94 & - & - \\
\hline Valor t-student ${ }^{\star \star}$ & \multicolumn{2}{|c|}{1.00} & \multicolumn{2}{|c|}{0.35} & \multicolumn{2}{|c|}{ - } \\
\hline Significancia (t-student) & \multicolumn{2}{|c|}{0.32} & \multicolumn{2}{|c|}{0.73} & \multicolumn{2}{|c|}{-} \\
\hline
\end{tabular}




\subsection{Opinión de participantes.}

La tabla $\vee$ resume los principales comentarios vertidos por los docentes durante la entrevista. Algunos comentarios se clasificaron como generales, mientras que otros se agruparon según la estrategia de interacción, comunicación o resolución de problemas.

Tabla V. Comentarios principales extraídos de las transcripciones de las entrevistas a docentes.

General:

- El uso de la tecnología es bueno para enseñar Estadística

o Considero que si mejoro el aprendizaje de los alumnos

- Las lecciones me crearon un ambiente de ayuda para la enseñanza

- Mejoró mi forma de enseñar Estadística, ya que los alumnos adquieren otra visión y me obligan a que la unidad se vea más a detalle

o Incluir tecnología web en cursos va a ser algo indispensable

- Para preparar mi curso, utilice mucho el web, ya que vienen muchos ejercicios

- La tecnología es un complemento importante, porque el alumno puede utilizar simuladores y herramientas didácticas

- El alumno puede tener acceso a más documentos y profundizar hasta donde le interese

- El alumno se dio cuenta que hay muchos apoyos y muchas maneras de aplicar la Estadística

o Contribuyo a mejorar su desempeño en el aula y en las evaluaciones

- La tecnología es una herramienta de apoyo

o El siguiente semestre lo voy a incluir

- En una semana revise un tema que normalmente requería de 2 semanas Interacción:

o La interacción es una estrategia adecuada

- Interactuar detrás del monitor les permite a los alumnos no ser tan tímidos y poder cuestionar

o La estrategia de interacción es básica para que el alumno no permanezca en forma estática

Comunicación:

o El chat y los foros crearon un ambiente de confianza y libertad entre el maestro y los alumnos

- La estrategia de comunicación fue la que más les gusto a los alumnos

o Hubo poca utilización de la estrategia de comunicación

Resolución de problemas:

- La resolución de problemas me pareció una estrategia interesante

- Con la resolución de problemas, a los alumnos les gustaba que en ese momento les decía si estaban bien o mal, era una especie de autoevaluación

- La estrategia de resolución de problemas es adecuada, sin embargo se requiere que los problemas sean más dirigidos al área o a la licenciatura objeto del curso

Por su parte, los alumnos que participaron en los grupos de discusión, expresaron sus comentarios a la pregunta expresa de ¿cuál estrategia consideraron más adecuada para la enseñanza de Estadística? Adicionalmente, se llevo un registro de la estrategia 
seleccionada y estar en condiciones de estimar un porcentaje. La tabla VI muestra el resumen de dichas opiniones.

Tabla VI. Opinión de alumnos acerca de la estrategia más adecuada para enseñar Estadística.

¿Cuál estrategia consideran más adecuada para la enseñanza de Estadística?

o El $39 \%$ de los alumnos consideró que las tres estrategias se complementan y son fundamentales para el aprendizaje

- El 36\% de los alumnos opinó que la resolución de problemas es la estrategia más adecuada para la enseñanza de Estadística

- El 18\% de los alumnos considera más adecuada la estrategia de interacción para la enseñanza de Estadística

- El 7\% de los participantes se inclina por la estrategia de comunicación para apoyar la enseñanza de Estadística. 


\section{Discusión}

Una reflexión que vale la pena comentar es que el constructivismo, como enfoque epistemológico, pretende dar una visión de cómo un sujeto se apropia de un conocimiento; sin embargo, no nos dice que procedimientos o instrucciones son óptimos para lograr que tal apropiación del conocimiento ocurra de la mejor manera. Considerando lo anterior, los resultados aquí presentados ilustran la factibilidad de utilizar algunos señalamientos teóricos del constructivismo para desarrollar objetos de aprendizaje en Estadística, específicamente aspectos teóricos relacionados con: i) la interacción del sujeto con determinados objetos, ii) la resolución de problemas en un contexto real y iii) el aprendizaje en comunidad.

Por otro lado, la organización de la información mediante el uso de objetos de aprendizaje, si bien presenta ventajas como: facilidad de manejo, transportabilidad, facilidad de reutilización, entre otras; también muestra desventajas. Se presentaron varios puntos de desacuerdo entre los docentes, específicamente en la delimitación y extensión de los temas. Además, el concepto de autonomía del OA, referida esta como la incorporación de la información necesaria para la enseñanza de un subtema en específico, requirió de un análisis más profundo y de revisiones y correcciones a los contenidos.

Con relación a las características generales de los participantes, los tres grupos presentan características relativamente homogéneas para variables tales como género, estado civil, actividad laboral y edad. Sin embargo, el grupo II muestra un mayor porcentaje, cercano al $40 \%$, de estudiantes que laboran, con una edad media mayor con relación a los otros dos grupos. Además, aproximadamente $20 \%$ de dichos estudiantes son casados. Estos factores pueden influir para que el grupo II tenga un promedio de aprovechamiento menor en universidad.

Un resultado interesante lo muestra la figura 4, ya que en 6 de 7 comparaciones entre los subgrupos de actividad mayor (ActMayor) versus actividad menor (ActMenor), el subgrupo con la mayor actividad con las lecciones tuvo un mejor resultado en su calificación media. Llama la atención los resultados encontrados con relación al mayor valor medio de calificaciones correspondientes al grupo de mayor actividad en el web. No obstante que dichas diferencias no fueron significativas (al nivel de significancia de 0.05), bien puede sugerir una mejoría en el aprendizaje de Estadística de los alumnos. 
Con relación a la opinión de los docentes acerca de las lecciones de Estadística y su efecto en su labor docente, los tres maestros coincidieron en señalar las bondades de la tecnología para apoyar las actividades didácticas. Consideraron a la tecnología un complemento importante en educación. Señalaron bondades para su quehacer docente como: ' ... crearon un ambiente de ayuda', ' ... me brindaron una nueva visión sobre las herramientas de apoyo en el web', '... pude recurrir a una gran cantidad de información estadística muy valiosa', '... ahora en una semana revise un tema que antes me tomaba dos semanas'. Todos coincidieron en que las lecciones propiciaron que el alumno mejorara su aprendizaje en la materia. Con relación a las estrategias utilizadas, hubo coincidencia en las bondades de cada una de ellas. Llamó la atención un comentario del docente del grupo II donde expresa que hubo poca utilización de la estrategia de comunicación. Esto coincidió con el desempeño académico menor que tuvo el subgrupo (ActMayor) en el tema de hipótesis.

A partir del intercambio personal de ideas que se dio con cada uno de los docentes participantes, hubo coincidencia de intereses y motivaciones para mejorar el intercambio de ideas y materiales entre ellos y de ser posible, conformar una comunidad de docentes interesados en la didáctica de Estadística.

Por su parte los alumnos señalaron las bondades de contar con una herramienta de apoyo como las lecciones de Estadística en línea. Hubo una gran cantidad de solicitudes para que se continúe con este tipo de herramientas en las otras materias. Los alumnos manifestaron que las tres estrategias (interacción, comunicación y resolución de problemas) son complementarias y que es difícil opinar sobre ellas de forma aislada.

Finalmente, es conveniente puntualizar las bondades de incorporar este tipo de herramientas de apoyo tanto a la didáctica del maestro como al aprendizaje de los alumnos. Los resultados de esta experiencia mostraron que si es posible mejorar el aprendizaje de los alumnos en Estadística a partir de propuestas didácticas innovadoras. 


\section{Referencias}

Álvarez-Gayou, J. J.L.A. (2005). Cómo hacer investigación cualitativa. Fundamentos y metodología. México: Paidós Educador.

Batanero, C. (2001). Didáctica de la Estadística. Granada, España: Grupo de Investigación en Educación Estadística. Departamento de Didáctica de la Matemática, Universidad de Granada.

Charles, C.M. (1988). Introduction to Educational Research. New York \& London: Longman Publishers.

Hodgins, W. (2000). Into the future. Recuperado el 15 de octubre del 2005, de http://www.learnativity.com/download/MP7.pdf

Leflore, D. (2000). Theory supporting design guidelines for web-based instruction. En Abbey, B. (Ed.), Instructional and cognitive impacts of web-based education (102-117). Hershey, PA: Idea Group Publishing.

Merrill, M.D. (2002). Second generation Instructional Design. Recuperado el 8 de enero de 2003, de http://www.id2.usu.edu/id2/index.htm

Mohan, P. (2004). Building an online course based on the e-learning standards; guidelines, issues, and challenges. Canadian Journal of Learning and Technology. 30 (3).

Murphy Elizabeth (2004). Moving from theory to practice in the design of web-based learning using a learning object approach. E-Journal of Instructional Sciencie and Technology. 7 (1). Recuperado el 8 de marzo del 2004 de http://www.usq.edu.au/electpub/e-jist/index.htm

O'Malley, J. (2002). Students Perceptions of Distance Learning, Online Learning and the Traditional Classroom. Online Journal of Distance Learning Administration, 2 (4). Disponible en: http://www.westga.edu/ distance/omalley24.html

Roig-Vila, R. (2005). Diseño de materiales curriculares electrónicos a través de objetos de aprendizaje. Recuperado el 15 de octubre del 2005, de http://www.um.es/ead/red/M4/roig42.pdf

Smith-Gratto, K. (2000). Strengthening learning on the web: Programmed Instruction and Constructivism. En Abbey, B. (Ed.), Instructional and cognitive impacts of webbased education (227-240). Hershey, PA: Idea Group Publishing.

Simonson, M., Smaldino, S., Albright, M. \& Zvacek, S. (2003). Teaching and learning at a distance. Foundations of distance education ( $2^{\text {nd }}$. ed.). Upper Saddle River, $\mathrm{NJ}$ : Prentice Hall, Inc.

Wiley, D.A. (2001). Connecting learning objects to instructional design theory: a definition, a metaphor, and a taxonomy. Utah State University. 